\title{
Innovating Method of Existing Mechanical Product Based on TRIZ Theory
}

\author{
Cunyou Zhao ${ }^{1}$, Dongyan $\mathrm{Shi}^{2,3}$, and $\mathrm{Han} \mathrm{Wu}^{3}$ \\ ${ }^{1}$ Mechanical Engineering College Heilongjiang Institute of science and technology, \\ Harbin 150027, China \\ ${ }^{2}$ Postdoctoral Research Station of Instrument Science and Technology, \\ Harbin University of Science and Technology, Harbin 150080, China \\ ${ }^{3}$ College of Mechanical and Electrical Engineering Harbin Engineering University, \\ Harbin 150001, China \\ \{Cunyou. Zhao, Dongyan. Shi , Han.Wu, cunyouzhao\} @sina.com
}

\begin{abstract}
Main way of product development is adaptive design and variant design based on existing product. In this paper, conceptual design frame and its flow model of innovating products is put forward through combining the methods of conceptual design and TRIZ theory. Process system model of innovating design that includes requirement analysis, total function analysis and decomposing, engineering problem analysis, finding solution of engineering problem and primarily design is constructed and this establishes the base for innovating design of existing product.
\end{abstract}

Keywords: TRIZ, conceptual design, innovating method, existing product.

\section{Introduction}

Although design is a creative act, absolute innovation is not existed from the influence of the design patterns to results of design. The design of mechanical products could be divided into two parts, of which one is original design and the other is adaptive design also called variant design. The first one is based on completely new working principle and new framework of structure, then former designs innovative mechanical products; while based on working principles and almost unchanged structural concept, the latter makes local adjustment of concrete structure according to functional requirements and designs adaptive conversion products.

In the process of practical design, about $70 \%$ designs are done following to adaptive design and variant design, which is reported by Pahl and Beitz [1]. Majority of the product design are from evolution of existing, so doing some researches on innovative design of mechanical products is urgent and of greatly practical importance in the process of design. Innovation design framework and innovation design thought are important for existing products of engineering. 


\section{Innovation Design Frameworks and Thought of Existing Product}

\subsection{Proposal of Innovation Design Framework of Existing Product}

\subsubsection{System Structure of TRIZ Theory and Innovation Flow}

TRIZ, as a methodology, can provide the process model, tools and methods for the designers in the conceptual design phase of mechanical design. Main research of TRIZ can be summed up in three categories: TRIZ theoretical basis, analytical tools and knowledge database. Structure system of its theory can be expressed as Fig. 1 [2].

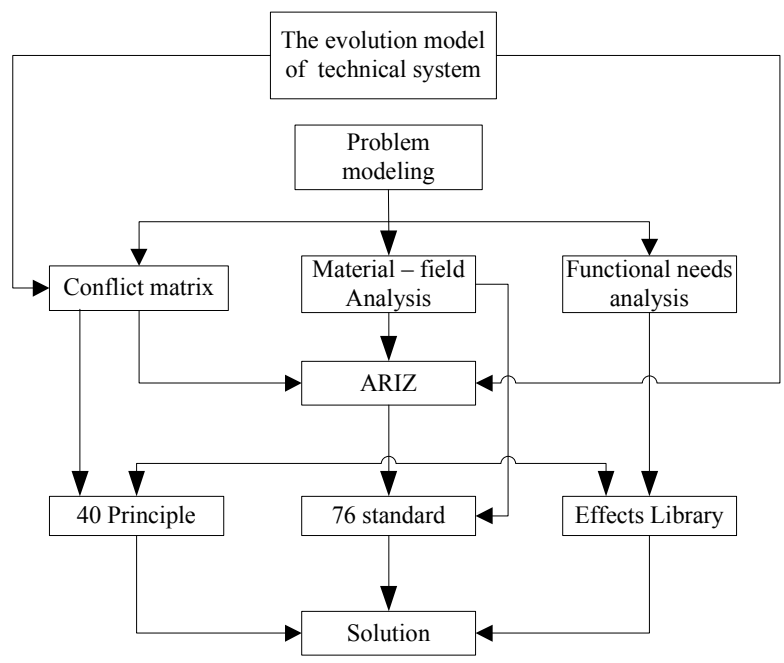

Fig. 1. Structure system of TRIZ theory

As shown in Fig. 1, TRIZ theoretical innovation process can be created in Fig. 2.

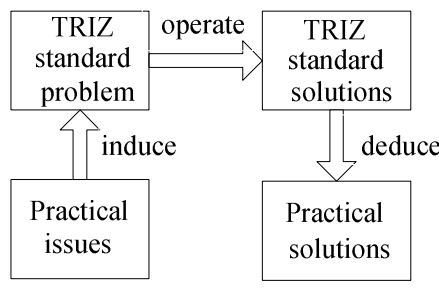

Fig. 2. Innovating flow of TRIZ

\subsubsection{Flow of Conceptual Design of Product}

There are analysis of user needs and problems of abstraction, function integration and analysis, design solution generation, evaluation of design solution and expression of solution in conceptual design. The general course of conceptual design is shown as 
Fig. 3. First of all, user requirements are standardized to design specification by designers, and then design specification is abstracted, to study out the total function of mechanical product. The total function will be divided into sub-functions which some principle solution respond layer by layer, which are to establish functional structure; and then sub-functions are solved by designers. Principle solution is integrated and evaluated. Finally, the principle solution [3] of the function is solved.

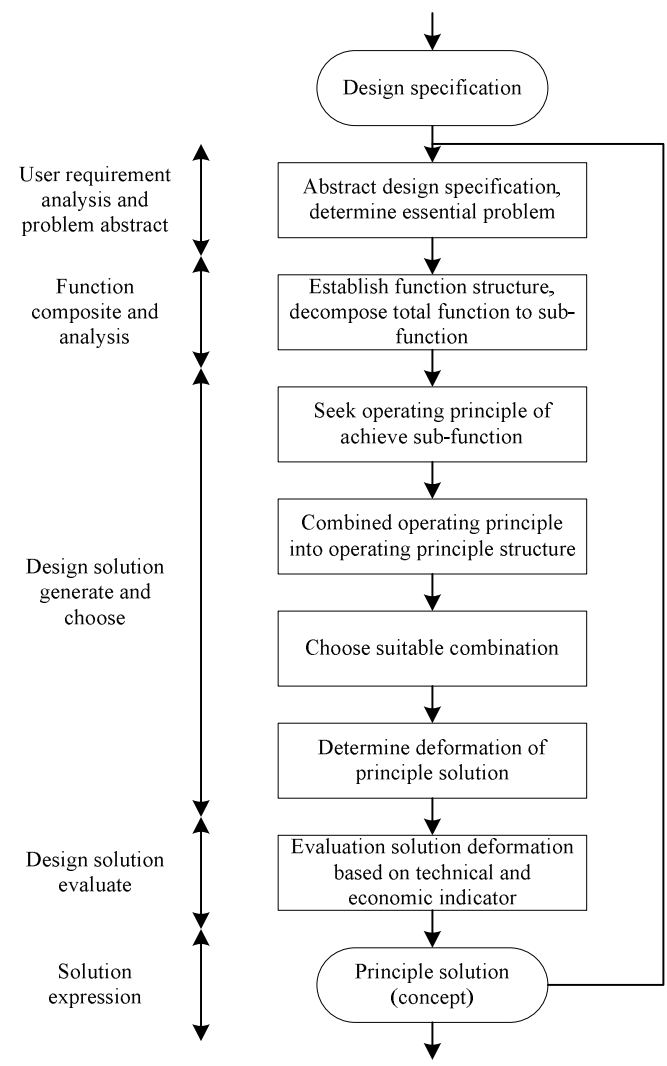

Fig. 3. Flow of conceptual design

\subsubsection{Innovation Design Framework of Existing Product}

As the change of requirement, existing mechanical products will not be satisfied with customer demand gradually, with requirement mapped to the mechanical products directly. The formation of function is reflected by the mechanical structure and work principle which have a mutual relationship. Innovative design framework is formed as shown in Fig. 4. It is divided into four layers of structures, demand domain, functional domain, principle domain and structure domain. There are mapping relations among functional domain, principle domain and structure domain, and there are mapping relations only between demand domain and functional domain. 


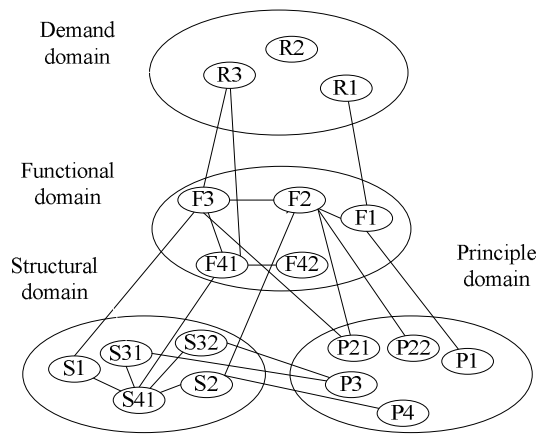

Fig. 4. Innovating design frame of existing product

\subsection{Innovation Design Thought of the Existing Mechanical Product}

Ideas of the existing mechanical products are put forward according to the TRIZ theory innovation flow in Fig. 2 and conceptual design flow in Fig. 3, as follows:

\subsubsection{Requirement Analysis}

When a new type of product is developed, a survey on market must be conducted with scientific methods. Then information about market demand and information of distribution is collected systematically and comprehensively. Uncertain factors and conditions in the future of market are predicted by qualitative analysis of experience or quantitative scientific computing and foundation is provided for product design of company.

\subsubsection{Analysis of the Total Function and Function Decomposition}

The function of product can be described with a black box which expresses the conversion flow of energy, materials and signal. It is also the relationship of input and output [4] when technology systems want to achieve a certain goal for the task. For the whole product, its function is transmission and transformation process of energy flow, material flow, information flow, the abstract description of effectiveness and capability.

The functions of existing products are familiarized and the pro-work of creative design is carried out, so that the function model is established. The general functional analysis of the product and functional decomposition enables designers to have a better understanding of the product and found the deficiency of product. Market demand can be met with better innovative design through comprehensive functional analysis and functional decomposition, engineering problems can be located in the functional level accurately.

\section{Description of total function}

The functional definition of study object, this definition should be accurate, concise and reasonably abstract, to seize the essence, to avoid the tendentious formulation. The description of the total function is the abstraction of product design task [5].

In engineering design, "black-box method" is one of the most frequently used abstract methods. Electro-mechanical system that need be designed is one "black box" 
which could not be seen the internal structure before solving. The relationship between input/output of designed system and outside environment is cleared by the "black box", which easily facilitate out of specific things while the functions of analysis and reflection. So it could enlighten designers to find a new better program [6] other than making sure a certain principle program too early. Relation between "Black box" and external conditions is shown in Fig. 5, the inner box is a mechanical system, the box is the border of the system, and the system is linked with the environment through the input and output of system.

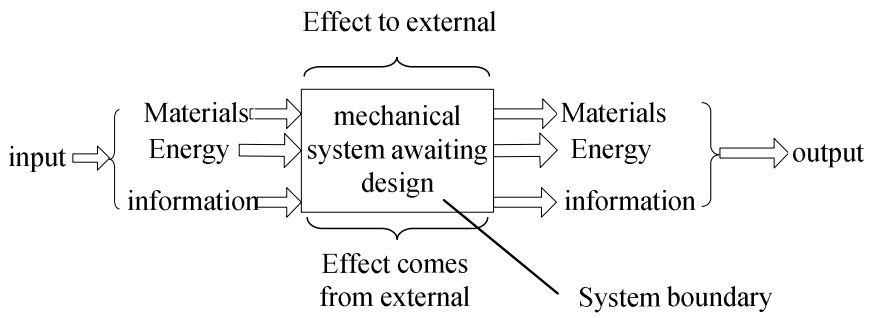

Fig. 5. Black box

\section{Function decomposition}

The whole function of the product is realized by various components which are assorted with each other, so the whole function, sub-function, function unit can be decomposed, simplified with the decomposition principles of systems engineering of the system, so as to achieve the solution of technical system [7] by assembling each function unit solution organically. Function can be decomposed to the function structure of the tree, which is known as function tree. Embryonic form of function model is established by considering the structure of upper sub-function. Then a perfect function structure chart is obtained by thinning the embryonic form layer by layer.

Decomposition of the system function should be integrated application of respective advantages of mechanical engineering and electronics and strive to constitute a simplified, modular system.

\subsubsection{Analysis of Engineering Problem}

Analysis of engineering problems is functional relation analysis, including analysis of the component model, analysis of the structure model and analysis of the function relation model. The purpose of analysis is to optimize the function of technology systems and realize the relationship between the consumption function.

1. Related conceptions and terms of TRIZ theory functional relation analysis According to the theory of TRIZ, the principle definition of functional relation analysis is defined with the related conceptions and terms as follows:

Technology systems: In order to meet the people (social) demands for integrated component of the material, a few systems can be integrated. In order to achieve a higher technical requirement, not only including technology systems, but also including labor organizations, production systems and control systems have to be considered. 
Components: Components are the parts of the technology system. The goal of analysis of the components is to study what are the system components. Here the components have generalized meaning, which not only refers to the parts, which is a part of the structure, and also are a few parts. The super-system components are ones which have interaction with technology system, in addition to the components of technology system. The components of system include components of technology system, components of super-system and effective object of system. A typical super-system consists of production equipment, storage and transport tools and the external environment.

Component model: The components of technology systems and the level of components are reflected by the component model. The approach of establishing the component model: find problems with the system (subsystem), set out the components of the system (subsystem) and its foundation of super-system, a list of its next level components.

Structure: Structure is the expression of the interaction between components. The goal of structural analysis is to study the relationship between the components.

Structure model: Structure model is the description of the relationship between the components, including the structure matrix and structure table. The approach of establishing the structure model: we need to establish the relationship between the components on the basis of component model, at the same time, describe relations with the components of external super-system, fill the structure table with marking the interaction.

Function: Function is the behavior of certain objects act on other objects and the parameters of other objects changed. The purpose of functional relation analysis is that the relationship between the components is transformed into components functional relation.

Functional relation model: Functional relation model is the description of the technical system existing various functions relation graph. So the customers understand the various functions of the components of the technical system, including useful function and harmful function.

\section{Functional relation analysis}

On the basis of total function description and function decomposition of machinery and electrical products, first of all, we locate customer requirement and design requirement in improving some sub-function, accessorial function or function unit of machinery and electrical products, that is the location of function requirement, will determine the need to solve engineering problems which is located in some corresponding sub-function, accessorial function or function unit, and then the components of the technical system which is corresponding to those functions are decided. The components of the technical the system are analyzed by functional relation analysis [8] with TRIZ theory to establish the component model, the structure model and functional relation model. The components include not only the components of the system or sub-system, but also the components of sub-systems related to the system or subsystem and effective objects. In this way, the interaction between the components, external environment, and effective objects are reflected by functional relation model of the component of the established technical system, various existing functions including useful and harmful functions can be understood, also the energy flow, information flow and harmful flow between the various components can be understood. 
On this basis, root cause analysis is carried out according to the user requirement which needs to be done. The analysis can be carried out not only from results to the cause, but also from the cause to the results so as to find the results of customer requirements or the reasons for demand works.

\subsubsection{Solution of Engineering Problem Creation Program}

With the evolution theory of TRIZ technology, there are four phases [9] in evolution process of machinery products: infancy, growth, maturity and exit. Mechanical products in growth is the composition form of most original technology system with improving the product on the basis of improve a function structure or principle function on the basis of the original mechanical product according to the market or customer demand.

According to the evolution of technology systems following the Ideal Final Result [10], the innovation design process of existing mechanical product is a scheme by improving the idealized level of products through mining available resources of product concept, to meet the market demand. Therefore, the status of available resources in improving product conceptual design became more important relatively, in that improved product is the short-term development of existing product, a large number of resources available which is existed in the existing products have not been used, the short-term development is mainly dependent on the utilization of these resources to promote.

Technical conflict, physical conflict, How-To model and substances- field model are defined by the results of the basic reason analysis, solved with innovative principles, separation principle, knowledge base and standard solution system respectively, and standard solution concept scheme of demand is established, and then innovation concept scheme of solving practical problem is formed by using the available resources of technology systems.

\section{Innovating Design Flow of Existing Product}

Function structure or working principle of existing products is changed correspondingly according to the requirement of improved design each time. Therefore, in the innovation design process, total functions re-analysis and function re-decomposition or corresponding changes according to demand on the basis of the structure analysis of original function should be done. On this basis, the sub-function, accessorial function or function unit of function structure are located accurately according to the market or customer demand, so as to the corresponding structural components of technology system of product structure are located specifically, and then the innovation concept scheme relative to function structure and product structure is analyzed and solved with the innovating flow of TRIZ theory[11-12].

On the basis of the total function analysis, the sub-function(accessorial function) or function unit of existing mechanical products relative to market demand products are found, so as to technology system of engineering problem is located accurately, then engineering problem is analyzed and solved according to the TRIZ theory with the main course: requirement analysis, total functional analysis, function decomposition, engineering problem analysis, engineering problem solving and preliminary design. The flow is shown in Fig. 6. 


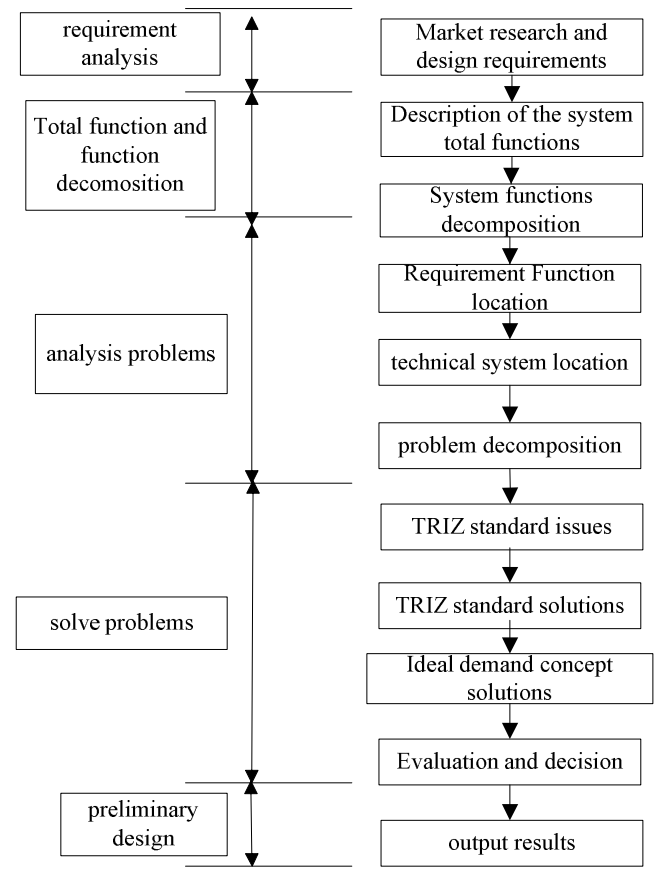

Fig. 6. Innovating design flow of existing product

\section{Conclusions}

The main way of product development is adaptive design and variant design based on existing product. In this paper, innovation design process of the existing product is proposed by combining the flow of conceptual design and innovating design flow of TRIZ, which lays a foundation for innovating design flow of existing product.

Acknowledgments. This work was supported by Natural Science Foundation of Heilongjiang Province of China No. E200819.

\section{References}

1. Pahl, G., Beitz, W.: Engineering Design. London: the design council, 186-188 (1984)

2. Altshuller, G.: And suddenly the inventor appeared, TRIZ, the Theory of Inventive Problem Solving. Massachusetts: Technical Innovation Center (1996)

3. Hsu, W., Irene Woon, M.Y.: Current research in conceptual design of mechanical products. Computer-Aided Design 30, 377-397 (1998)

4. Yoshikawa, H.: Design Philosophy. The state of the art. Annals of the GIRP 38 (1989)

5. Li, J.: Electromechanical Integration Technology, pp. 102-104. Science Press, Beijing (2004) 
6. Umeda, Y., Ishii, M., Yoshioka, Y.M., et al.: Supporting Conceptual Design Based on the Function-Behavior-State Modeler. Artificial Intelligence for Engineering Design, Analysis and Manufacturing, 275-288 (1996)

7. Han, X.: Function design method. Invention and Reform 11 (2001)

8. Tan, R., Yuan, C., Cao, G., Zhang, R.: Function model for products existed using reverse fishbone. Journal of Engineering Design 8, 197-201 (2003)

9. Gahide, S.: Application of TRIZ to Technology Forecasting Case Study: Yarn Spinning Technology. TRIZ Journal (2000)

10. Domb, E.: The Ideal Final Result: A Tutorial. The TRIZ Journal (February 1997), http: / / www. TRIZJournal.com/

11. Donlin, M., Chinghsue, C., Jiaanchern, L.: Evaluating Weapon System Using Fuzzy Analytic Hierarchy Process Based on Entropy Weight. Fuzzy Set and Systems 62, 127-134 (1994)

12. Hague, M.J., Taleb-Bendiab, A.: Tool for the management of concurrent conceptual engineering design. Concurrent Engineering, 111-112 (1998) 